EOMmun: Communication et organisation

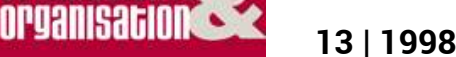

Management par projet et logiques

communicationnelles

\title{
Communication et gestion des conflits dans les projets
}

\section{Rolande Marciniak}

\section{OpenEdition}

\section{Journals}

Édition électronique

URL : http://journals.openedition.org/communicationorganisation/2028

DOI : 10.4000/communicationorganisation.2028

ISSN : 1775-3546

Éditeur

Presses universitaires de Bordeaux

Édition imprimée

Date de publication : 1 mai 1998

ISSN : 1168-5549

Référence électronique

Rolande Marciniak, "Communication et gestion des conflits dans les projets », Communication et organisation [En ligne], 13 | 1998, mis en ligne le 26 mars 2012, consulté le 19 avril 2019. URL : http:// journals.openedition.org/communicationorganisation/2028 ; DOI : 10.4000/

communicationorganisation. 2028

Ce document a été généré automatiquement le 19 avril 2019

(C) Presses universitaires de Bordeaux 


\title{
Communication et gestion des conflits dans les projets
}

\author{
Rolande Marciniak
}

1 Selon la norme X-50-105 (Afnor, 1994), un projet se définit comme une démarche spécifique, qui permet de structurer méthodiquement et progressivement une réalité à venir. Un projet est caractérisé tout d'abord par la satisfaction d'un besoin spécifique et particulier, ensuite par un objectif autonome, en ce sens que tout projet a un début et une fin, enfin par un caractère novateur, au moins partiellement (technique, géographique, organisationnel, etc.).

2 Les professionnels ont proposé des méthodes pour mieux gérer les projets. Très succinctement elles visent soit à estimer (les délais, les coûts, les gains attendus...) soit à conduire les activités de projet. Ces méthodes, en évolution permanente, constituent, au mieux, des conditions nécessaires mais pas suffisantes de la réussite des projets informatiques.

3 Les recherches menées par le monde académique ont vu, pendant longtemps, s'affronter deux approches des processus organisationnels et des projets: le déterminisme organisationnel et le déterminisme technologique. Les résultats de ces études déterministes s'étant avérés contradictoires et ambigus, une approche, privilégiant l'interaction dynamique des facteurs, remet en cause ces fondements déterministes. D'une part, selon cette approche, le comportement des acteurs participant directement ou indirectement à un projet ne peut pas être prédit de manière certaine, aussi le déterminisme organisationnel rencontre-t-il de sérieuses limites d'applicabilité. D'autre part, il existe une imprévisibilité relative de la technique. Aussi les tenants de l'interaction dynamique jugent-ils le déterminisme technologique insuffisant pour expliquer les résultats d'un projet. Ce processus est le résultat d'interactions complexes et imprévisibles entre des contraintes externes liées à la technologie, des facteurs structurels et des motivations ou rationalités individuelles. Aussi la gestion des conflits constitue-t-elle un aspect primordial du processus projet, véritable pivot autour duquel se 
déroulent les activités, se manifestent les contraintes, se prennent les décisions, se déploient les logiques d'action et finalement émergent les finalités du projet.

Dans une première partie, cet article décrit la complexité des projets en structurant des concepts dans un modèle théorique. La deuxième partie examine comment la gestion des conflits constitue un pivot de ce modèle.

\section{La complexité des projets informatiques}

5 Les ingrédients de la pensée complexe se prêtent mal au découpage analytique, notamment du fait de leur imbrication mutuelle. Aussi, présenterons-nous tout d'abord les concepts utilisés, selon la séquence de leur apparition dans le processus de création de notre modèle, dont la source d'inspiration provient des épistémologies de la pensée complexe (Morin, 1990). Nous pourrons ensuite développer l'argumentation relative au choix de ces concepts pour l'étude des processus d'informatisation.

\section{Genèse du modèle de la complexité des projets informatiques}

La première notion à laquelle nous nous sommes attachés est celle de mutidimensionnalité, dont trois directions ont été poursuivies: multi dimensionnalité des résultats, des acteurs, et des processus d'un projet. Chacune de ces trois directions a été investiguée. Chemin faisant, la réflexion faisait émerger d'autres concepts ou notions, dont on argumentera plus loin l'intérêt pour notre champ de recherche. Nous nous attacherons dans cette partie à les énumérer.

7 Tout d'abord, la multi dimensionnalité des résultats d'un projet nous a conduite à remonter au concept de l'efficacité organisationnelle et à en déduire les implications essentielles pour le contexte de notre étude ; la notion complémentaire de paradoxe s'est avérée absolument nécessaire à la compréhension du concept d'efficacité. L'aboutissement de cette direction de la multi dimensionnalité a consisté à définir différents critères de la réussite d'un projet, et à signaler la variété des variables permettant d'aboutir à cette réussite multidimensionnelle.

Ensuite, la multiplicité des processus ${ }^{1}$ implique des découpages (sous-systèmes) du projet mais aussi des unités (autres systèmes) dans lesquelles il "s'insère ». Les critères de découpage d'un projet sont variés: le temps (découpage en phases séquentielles remis partiellement en cause par l'ingénierie simultanée), les activités (découpage selon les compétences spécifiques requises), le produit (découpage en lots fonctionnels), etc. Pour cette deuxième direction de la multi dimensionnalité, nous avons adopté la perspective systémique d'imbrication de systèmes ${ }^{2}$. Les processus constituent ainsi les variables de compréhensibilité du modèle, et leur enchaînement temporel en fonde la dynamique.

9 Enfin, la multiplicité et la variété des acteurs se manifestent inévitablement par des rationalités (perceptions, inférences, évaluations...) différentes, pouvant diverger voire s'opposer, sur le déroulement et les issues d'un projet informatique. Aussi cet aspect de la multi dimensionnalité nous a-t-il dirigé vers la problématique des rationalités des acteurs. Cette réflexion aboutit à accorder une place centrale aux conflits survenant dans les projets informatiques, et à leurs différents modes de résolution.

10 La genèse du modèle ${ }^{3}$ vient d'être brossée à grands traits, nous allons maintenant reprendre, pour argumenter nos choix, chacun des points suivants : 
11 1. multi dimensionnalité et paradoxes de l'efficacité des projets,

12 2. la temporalité, l'imbrication de processus et l'écosystème des projets.

\section{Multi dimensionnalité et paradoxes de l'efficacité}

13 Le concept d'efficacité organisationnelle ne peut pas être ignoré en dépit de l'ambiguïté qui l'entoure. Empiriquement il demeure l'ultime variable dépendante de toute recherche. Au niveau de l'action, les différentes parties prenantes d'une organisation, clients, fournisseurs, salariés, dirigeants, propriétaires..., sont continuellement confrontées au problème de son évaluation. La multi dimensionnalité de ce concept a été démontrée et validée par de nombreuses recherches (Montebello, 1976, Quinn \& Rohrbaugh, 1983).

14 L'efficience est une notion qui recouvre l'aptitude à minimiser les coûts de transformation d'intrants en produits acceptables. Selon le point de vue adopté, quant aux résultats, trois catégories d'efficience sont distinguées: économique, sociale et technique ; chacune d'entre elles est mesurée par des critères spécifiques :

15 1. L'efficience économique compare des intrants et des extrants en termes de coûts. Lorsque la quantification des gains est possible, l'efficience économique du projet est obtenue par le calcul des ratios de choix d'investissement.

16 2. L'efficience sociale est définie par l'intégration, l'implication et l'atteinte des objectifs personnels de chaque membre de l'organisation. En ce qui concerne les projets on doit distinguer l'efficience sociale du projet et celle du produit (ou de l'ouvrage) utilisé (ou exploité) à l'issue du projet. La première concerne le point de vue des membres de l'équipe, dans quelle mesure le projet a constitué une expérience professionnelle enrichissante et motivante. La seconde se place du point de vue des usagers et des utilisateurs, dans quelle mesure l'utilisation de l'ouvrage ou du produit contribue à l'amélioration de leurs conditions de travail ou d'existence.

17 L'efficience technique est définie comme l'aptitude à produire des biens en quantité et qualité acceptable. Pour notre propos il s'agira de la qualité de l'ouvrage ou du produit en termes de fiabilité, de performance, maintenance...

18 Une autre facette de l'efficacité, la capacité à atteindre des objectifs ou "l'effectivité ", fait référence à des estimations préalables, à des buts prédéfinis. Il s'agit alors des gains obtenus, comparés aux gains attendus, mais aussi de la réalisation du projet dans des limites temporelles et financières préalablement définies.

Des études ont démontré le caractère paradoxal du concept d'efficacité (Cameron, 1986, Lewin \& Minton, 1986). L'efficacité est ainsi perçue de manière différente selon les individus. Le consensus sur un ensemble suffisant de critères est impossible à atteindre dans la mesure où les valeurs et les préférences individuelles sont différentes voire contradictoires, difficiles à identifier par les individus eux-mêmes, et variables selon le temps. Les réussites (versus échecs) d'un projet pourront donc être perçues différemment selon que l'on est exploitant, utilisateur final ou usager, chef de projet, ou membre de l'équipe de projet, maître d'œuvre, client ou maître d'ouvrage...

Le paradoxe provient aussi du fait qu'une organisation efficace doit posséder des attributs variés, contradictoires, voire mutuellement exclusifs et opérant simultanément, tout en 
ne se renforçant pas. Ainsi en est-il du respect des délais qui peut entrer en conflit avec la qualité du produit et l'efficience sociale du projet.

On peut donc s'attendre à l'émergence de paradoxes dont on devra tenter l'interprétation : des variables déterminantes différentes selon les indicateurs d'efficacité, l'intervention contradictoire d'une même variable déterminante, le rôle non linéaire d'un prédicateur dont l'intensité provoque, à un certain seuil, l'inversion du sens de son intervention.

\section{La flèche du temps, les processus et l'écosystème ${ }^{4}$ des projets}

22 L'approche complexe implique l'inclusion du facteur temps pour interpréter le déroulement d'un projet et en comprendre l'aboutissement, a priori relativement imprévisible. Dès lors, il convient de noter que les voies méthodologiques privilégiées sont l'étude longitudinale et la recherche-action.

En ce qui concerne les processus, une option aurait pu consister à distinguer un processus par phase de projet. Cette option a cependant plusieurs inconvénients. Tout d'abord les pratiques de découpage varient, selon les méthodes et les outils mis en œuvre ou les secteurs industriels, engendrant une polysémie difficile à maîtriser; de plus se pose le problème de l'agrégation de processus qui peuvent avoir été menés soit en séquence, modèle traditionnel de la cascade, soit en concomitance relative, modèle de l'ingénierie simultanée. Une autre option consiste à distinguer deux processus, le processus de fonctionnement interne de l'équipe de projet, et le processus d'interaction avec les autres parties prenantes du projet. La deuxième option, outre qu'elle ne possède pas les inconvénients de l'option par phases, répond mieux au critère d'imbrication de systèmes que nous avons évoqué plus haut, dans la mesure où le système « autres parties prenantes du projet » constitue l'environnement immédiat du système « équipe de projet ».

La prise en compte de l'écosystème du projet, amorcée par le découpage vu précédemment, doit être poursuivie par le repérage de trois catégories pertinentes de l'environnement d'un projet. En effet un projet, quel que soit son degré d'autonomie, n'est jamais complètement isolé. Le projet peut, par ses effets, modifier les structures de l'organisation qui l'abrite, et ces effets sont mesurés, dans le modèle, par les indicateurs d'efficacité-efficience cités plus haut. Mais il peut aussi subir l'influence des structures de l'organisation dans laquelle il se déploie. Tout d'abord, l'étude de faisabilité constitue l'environnement temporel précédant le lancement du projet. Enfin deux types d'environnement fonctionnel sont à prendre en compte, d'une part l'unité ou les unités qui abrite(nt) le projet: le maître d'œuvre interne ou externe, le ou les Co (sous)traitants, d'autre part l'unité (ou les unités) qui exploiteront (utiliseront) l'ouvrage (le produit) : le maître d'ouvrage, le client, l'exploitant... La façon dont sont conduites les activités, et plus particulièrement les projets, dans ces différents environnements composent le climat général dans lequel va baigner le projet.

L'ensemble de ces cinq processus (activités internes de l'équipe de projet, d'interaction avec les autres partie prenantes du projet, d'étude préalable, des unités environnantes) constitue dans le modèle le groupe des variables indépendantes qui auront un impact sur les résultats du projets, c'est-à-dire sur les indicateurs d'efficacité-efficience cités plus haut. Comme on l'a souligné précédemment (cf. le paradigme de l'efficacité) ces effets peuvent être paradoxaux, et relativisent l'aspect déterministe du modèle ${ }^{5}$. Ainsi conçue, l'imbrication des processus du déroulement des projets informatiques nous conduit à 
analyser l'enchaînement (par antériorité) des processus ; cette analyse permet d'affiner la compréhension du déroulement des projets, et de valider le découpage (en processus) du modèle et le rôle pivot de la gestion des conflits.

\section{La gestion des conflits conductrice et réductrice de la réussite des projets : une illustration par les projets informatiques}

Nous accordons un rôle primordial aux modes de gestion des conflits, dans la mesure où ils servent à véhiculer l'imprévisibilité et contribuent à la gérer plus ou moins efficacement.

\section{Des rationalités différentes et des conflits inévitables}

Les projets informatiques se composent d'activités impliquant la mise en œuvre de savoir-faire techniques spécifiques et requièrent des compétences variées, c'est-à-dire qu'ils nécessitent l'intervention et la coordination d'acteurs multiples. Or cette différenciation organisationnelle des participants d'un projet, verticale ou hiérarchique, horizontale ou division sociale du travail, mais aussi contractuelle dans le cas du développement externe, a une incidence sur l'émergence des désaccords. En effet, selon leur position, les acteurs ont accès à des informations différentes et de ce fait sont conduits à interpréter distinctement les problèmes posés, et à envisager des solutions qui peuvent être divergentes. La différenciation organisationnelle constitue donc une source potentielle importante des conflits.

La réalisation des différentes tâches implique une forte interdépendance des acteurs ; or celle-ci peut donner lieu à des perceptions de manœuvres d'obstruction, réelles ou imaginaires, c'est le cas notamment lorsque les informaticiens se plaignent de leur difficulté à obtenir des informations de la part des responsables utilisateurs.

De plus, le partage de ressources limitées contribue à renforcer l'incompatibilité des objectifs et à encourager les rivalités. On rencontre fréquemment ce phénomène entre les différentes équipes informatiques dans l'utilisation des matériels de tests ou à propos de l'affectation des individus sur les projets.

Enfin les projets informatiques sont par essence un nid de fécondation des dissensions dans les entreprises, non seulement parce qu'ils concernent les systèmes d'information des organisations et que la détention des ressources informationnelles peut constituer un enjeu de pouvoir, mais aussi parce que ces projets sont souvent porteurs de changements organisationnels importants.

31 Ainsi la différenciation organisationnelle, le partage de ressources limitées, l'interdépendance des tâches qui caractérisent les projets, et la nature même de l'informatisation, conduisent à des impressions d'incompatibilité et d'entrave, et ces perceptions aboutissent à des situations conflictuelles. 


\section{L'impact des formes et modes de résolution des conflits} d'une même dimension par le truchement d'une autre dimension. Ainsi un mode 
d'expression irrationnel, canalisé par une structure formelle adaptée, facilitateur compétent par exemple, pourra ensuite s'exprimer rationnellement. Un conflit privé, qui ne peut pas s'exprimer au travers de structures formelles, pourra devenir public en utilisant des relations informelles. Les mécanismes par lesquels les conflits sont contenus, canalisés ou résolus orientent leur aspect constructif ou au contraire destructeur. Les conflits peuvent être positifs dans la mesure où ils focalisent l'attention sur des problèmes importants et sur la nécessité de les résoudre, incitent à la créativité et à l'innovation, stimulent l'intérêt et la motivation et augmentent la cohésion des groupes. Mais a contrario ils peuvent être négatifs lorsqu'ils favorisent l'hostilité, l'obstruction et l'aliénation et lorsqu'ils dissipent les énergies.

Constructeurs ou destructeurs, les modes de résolution des désaccords ont un effet sur les résultats des projets. De ce fait, l'analyse de la communication entre les différentes parties prenantes du projet est essentielle.

38 La nature de l'interaction d'un acteur indique la manière selon laquelle il aborde une situation conflictuelle. Une interaction détachée indique un acteur indifférent aux enjeux, par inconscience ou par crainte; il y a adoption d'une attitude de retrait, indifférence vis-à-vis de ses propres aspirations. En général le pouvoir détenu est faible. Il se peut aussi que les enjeux soient particulièrement faibles pour l'acteur. Lorsque la nature de l'interaction est accommodante, les enjeux sont perçus et il y une prédisposition à « ne pas faire de vagues » et à céder du terrain. La nature compétitive de l'interaction indique que les enjeux sont perçus importants et que l'acteur veut imposer son point de vue. Enfin l'interaction coopérative signale une volonté de comprendre la situation et les enjeux, ainsi qu'une orientation vers l'intercompréhension. Or les modes de résolution des conflits (Van de Ven \& Ferry, 1980) vont dépendre de la nature des interactions déployées par les protagonistes d'un conflit.

39 Ainsi, lorsque le détachement domine, les conflits sont ignorés. Le résultat de ce mode de «résolution» est en général insatisfaisant, et peut conduire à des impasses catastrophiques en phase opérationnelle, c'est à dire à la mise au rebut du logiciel réalisé. L'interaction coopérative exprime le désir des acteurs de réaliser, par la confrontation de leurs désaccords, l'intégration de leurs aspirations. Les aspirations de départ peuvent ne pas être totalement intégrées, cependant les compromis réalisés seront communément acceptés. L'issue donne donc satisfaction aux parties même si elles ont été conduites à revoir leurs aspirations originelles. La confrontation des points de vue, parce qu'elle exige conviction et ténacité des acteurs, permet une intégration sélective et efficace des objectifs. Non seulement le mode de résolution des conflits par confrontation favorise l'efficacité organisationnelle, mais il permet aussi d'atteindre l'efficience sociale du projet ; en effet l'intercompréhension, nécessaire à la confrontation, constitue un facteur de développement des membres de l'équipe de projet.

40 La nature compétitive est fondée sur le désir de satisfaire ses propres aspirations aux dépens des autres; les conflits sont déclarés. Une confrontation préalable peut avoir eu lieu qui n'a pas abouti au consensus, l'orientation compétitive domine et les désaccords doivent alors être résolus en recourant à la voie hiérarchique. Le résultat de ce mode de résolution peut être un compromis qui satisfait ou non les parties, ou bien une décision tranchée qui ne satisfera pas l'une des parties au moins.

41 La nature accommodante, lorsqu'elle domine l'interaction, conduit à minimiser et à atténuer l'amplitude des désaccords pour " ne pas faire de vagues ", c'est-à-dire pour éviter le recours hiérarchique et/ou la confrontation. Lorsque les acteurs disposent 
de ressources, les tractations et marchandages constituent les fondements de ce mode de résolution. Mais l'origine de ce mode de résolution peut aussi être culturelle, tradition de réticence à la confrontation, organisationnelle, absence de règles de recours, faiblesse du management hiérarchique, différence de pouvoir entre les parties. L'issue de ce mode de résolution peut ou non satisfaire les parties.

Or les conflits peuvent surgir au cours des différentes étapes d'un projet. Aussi convient-il d'examiner les différents processus propres à les héberger. Le recours à la voie hiérarchique, ainsi que l'atténuation des conflits ont des effets paradoxaux. La fréquence plus élevée du mode hiérarchique est vraisemblablement liée à l'existence de règles formelles et procédures de recours en cas de désaccords.

\section{Processus : les cercles vicieux et vertueux des projets}

La combinaison des relations, entre les variables-clés, c'est-à-dire la structure dynamique du système projet, dévoile des processus amplificateurs, bénéfiques ou néfastes (Senge, 1991). Pour chaque dimension de la réussite des projets on peut découvrir deux circuits l'un vertueux, l'autre vicieux.

44 L'examen de chacun de ces circuits aide à comprendre la logique sous-jacente, à déceler les causes structurelles et leur interaction avec des causes factuelles ou apparentes. Le propos sera illustré avec l'exemple des gains organisationnels engendrés par le nouveau système informatique: réductions de coût, gains directs, avantages qualitatifs, concurrentiels...

Le cercle vertueux (cf. figure 1) est engendré par la satisfaction des utilisateurs à l'égard des systèmes d'information préexistants au nouveau logiciel ; cet état de grâce engendre des méthodes de travail entre informaticiens et non informaticiens caractérisées par la confrontation à propos des choix fonctionnels et d'organisation et la participation active de toutes les parties prenantes. L'ensemble de ces éléments constitue un nœud de causalité structurelle, dont on ne peut, à vrai dire, dégager l'origine. Est-ce parce que les utilisateurs sont satisfaits que ces méthodes de travail sont adoptées? Ou bien est-ce l'utilisation, à l'origine, de ces méthodes de travail qui a provoqué la satisfaction? Il y a là un exemple de circuit spirale dans lequel les effets sont nécessaires au processus qui les génère. Par ailleurs, on décèle une cause plus factuelle, relative à la composition de l'équipe de projet. Lorsque les membres de l'équipe ont précédemment acquis de l'expérience dans leur fonction sur le projet (chef de projet, concepteurs, réalisateur), leur compétence dans les activités de validation favorise la réussite. 


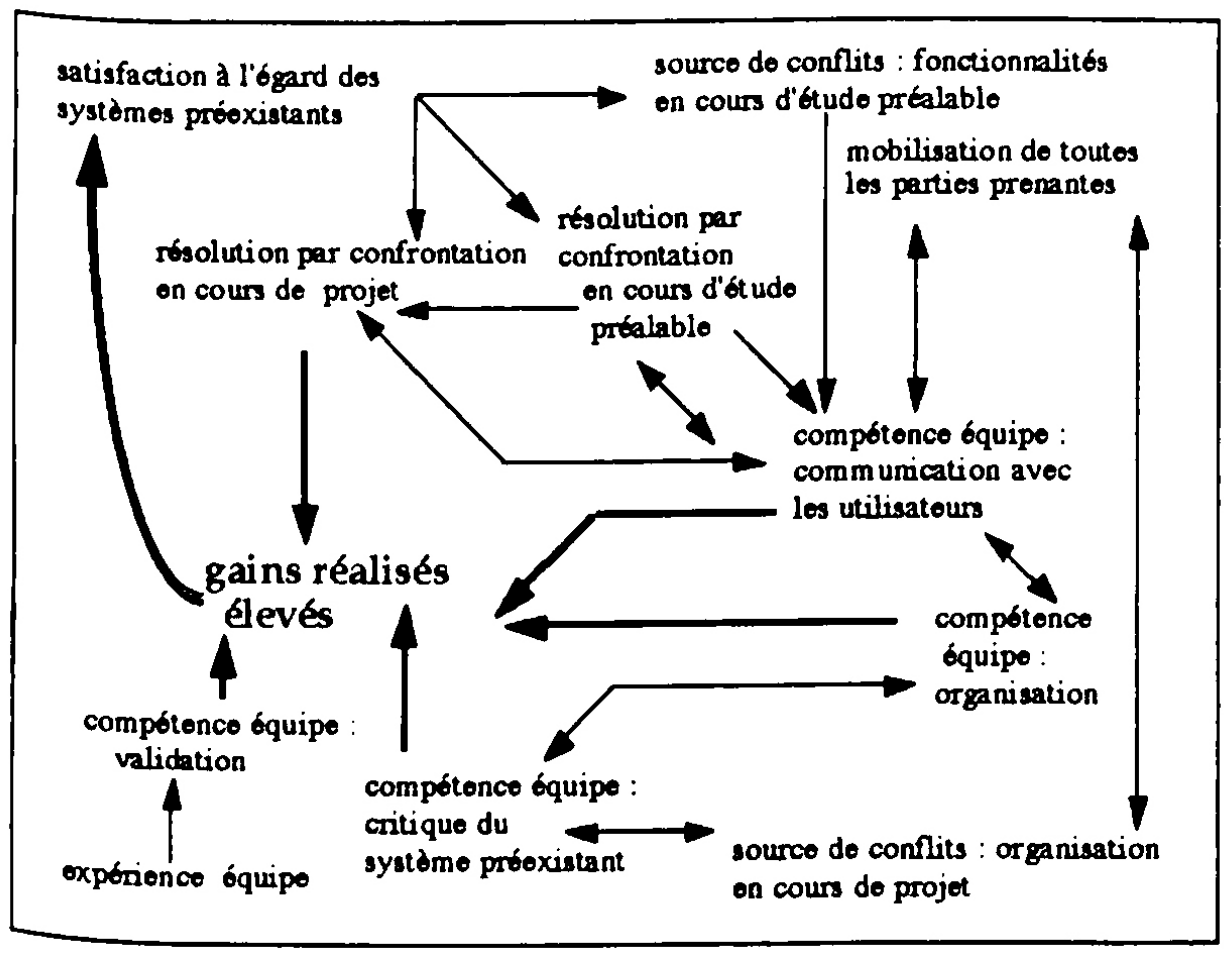

Dans le cas du cercle vicieux (cf. figure 2), on découvre deux nœuds de causalité structurelle. Le premier circuit est en quelque sorte l'inverse du cas précédent, c'est-àdire qu'une des causes originelles concerne l'insatisfaction des utilisateurs à l'égard de leurs systèmes d'information. Cependant leur attitude a priori très favorable à l'égard de l'informatique complète ce circuit. Les données disponibles n'ont pas permis de vérifier si ces utilisateurs sont plus technicistes que les techniciens et donc utopistes ou simplement avertis et donc virulents à l'égard de l'impéritie des informaticiens. En réalité les deux cas de figures peuvent exister. Le deuxième nœud de causalité structurelle concerne les structures du département informatique, plus spécifiquement son acculturation à travers des modes spécifiques de résolution des conflits qui vont se transmettre dans les projets. 


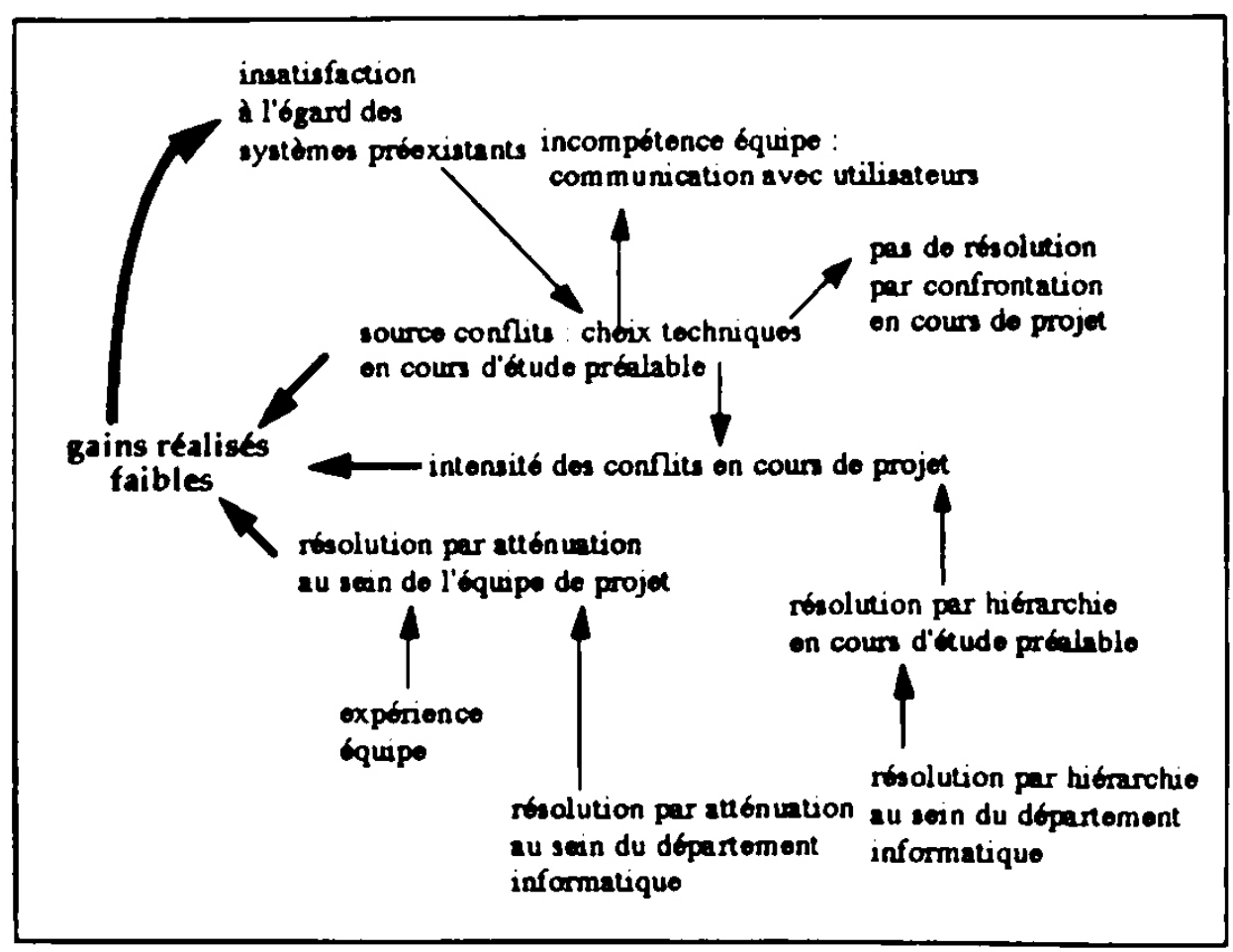

Notons que la même cause factuelle, l'expérience de l'équipe intervient ici négativement et illustre le caractère paradoxal de l'efficacité. Peut-on interpréter ce paradoxe ?

D'un côté, cercle vertueux, l'expérience de l'équipe lui permet de réaliser ses activités de validation de manière efficace.

De l'autre côté, cercle vicieux, l'équipe aura tendance, du fait de son expérience (dans le cas du cercle vicieux il s'agit d'une expérience multiple: fonction, domaine applicatif, informatique), à régler les problèmes rencontrés par ajustements internes au lieu de se confronter avec les autres parties prenantes du projet, notamment les utilisateurs et les facilitateurs. De ce fait l'éventail, plus restreint, des réponses apportées conduit à des solutions engendrant des gains organisationnels plus faibles.

Il convient de noter aussi que l'activation d'abord positive, puis négative, de la variable « expérience de l'équipe » confirme notre argumentation précédente à propos du « ... rôle non linéaire d'un prédicteur dont l'intensité provoque, à un certain seuil, l'inversion du sens de son intervention."

51 L'élaboration de diagrammes systémiques bipolaires pour chacune des variables dépendantes, c'est-à-dire pour chaque dimension de la réussite et de l'échec, a une vertu heuristique puisqu'elle permet, à partir de fondements théoriques sans cesse améliorés, de découvrir les logiques sous-jacentes du fonctionnement des projets.

\section{Conclusion}

52 L'étude a montré les effets bénéfiques du recours à la confrontation des désaccords, et ce constat rejoint les résultats d'études antérieures réalisées sur d'autres projets que les projets informatiques (Kezsbom Schilling \& Edward, 1989). Cependant, compte tenu du 
contexte dans lequel émerge le conflit (le pouvoir détenu par chacune des parties prenantes, la philosophie personnelle de la personne qui doit gérer le conflit, l'impact du mode de résolution du conflit sur les délais, le budget, les équipes, les conditions structurelles préexistantes au projet...) des stratégies circonstanciées et dynamiques pourraient être adoptées. Le tableau 1 décrit ces stratégies.

Des mécanismes peuvent être mis en place dans les organisations pour favoriser un mode de résolution particulier.

Par exemple, on fait intervenir des médiateurs pour permettre une expression plus affirmée des parties les plus faibles et favoriser la confrontation, notamment par des transitions sur les dimensions bipolaires des conflits. Ou bien dans d'autres organisations on définit des règles de recours hiérarchique pour résoudre les conflits non résolus aux niveaux inférieurs. On peut aussi mettre en place des mesures préventives afin de diminuer les occasions de conflits. Ainsi la vérification systématique et approfondie des contrats de sous-traitance avant leur signature permet d'en corriger les termes ambigus, sources courantes d'interprétations divergentes et, par amplification, de conflits.

Tableau 1. La contingence des modes de résolution des conflits

\begin{tabular}{|c|c|c|}
\hline $\begin{array}{c}\text { Mode } \\
\text { de résolution }\end{array}$ & $\begin{array}{l}\text { Conseillé } \\
\text { quand }\end{array}$ & $\begin{array}{c}\text { Déconseillé } \\
\text { quand }\end{array}$ \\
\hline $\begin{array}{l}\text { - ignorer les } \\
\text { désaccords } \\
\text { - conflits occultés } \\
\text { - résistance passive }\end{array}$ & $\begin{array}{l}\text { - l'enjeu est peu } \\
\text { important } \\
\text { - l'enjeu tend à s'effacer } \\
\text { - solution temporaire }\end{array}$ & $\begin{array}{l}\text { - l'enjeu est important } \\
\text { - l'enjeu est persistant }\end{array}$ \\
\hline $\begin{array}{l}\text { - atténuer les } \\
\text { divergences de points } \\
\text { de vue } \\
\text { - ne pas faire de } \\
\text { vagues } \\
\text { - adopter un } \\
\text { compromis }\end{array}$ & $\begin{array}{l}\text { - comme ci-dessus plus } \\
\text { - relations feutrées } \\
\text { nécessaires } \\
\text { momentanément } \\
\text { - chaque partie a } \\
\text { quelque chose a offrir } \\
\text { - les ressources sont } \\
\text { limitees. }\end{array}$ & $\begin{array}{l}\text { - comme ci-dessus plus } \\
\text { - la solution est } \\
\text { irréaliste } \\
\text { - l'engagement des } \\
\text { partenaires semble } \\
\text { douteux } \\
\text { - les parties sont } \\
\text { disposées a la } \\
\text { confrontation }\end{array}$ \\
\hline $\begin{array}{l}\text { - combattre les autres } \\
\text { points de vue } \\
\text { divergents } \\
\text { - recours hiérarchique } \\
\text { - coup de force }\end{array}$ & $\begin{array}{l}\text { - des règles et } \\
\text { procédures sont en } \\
\text { vigueur } \\
\text { - le pouvoir est réel du } \\
\text { fait de la position ou } \\
\text { de l'autorité exercée }\end{array}$ & $\begin{array}{l}\text { - les perdants n'ont pas } \\
\text { la possibilité d'expri- } \\
\text { mer leurs besoins } \\
\text { - il y a un risque d'ef- } \\
\text { fondrement futur } \\
\text { notamment du fait de } \\
\text { l'instabilité du } \\
\text { pouvoir }\end{array}$ \\
\hline $\begin{array}{l}\text { - confronter les points } \\
\text { de vue divergents } \\
\text { - sincérité } \\
\text { - écoute }\end{array}$ & $\begin{array}{l}\text { - du temps est } \\
\text { disponible pour la } \\
\text { confrontation } \\
\text { - les parties prenantes } \\
\text { sont aptes et } \\
\text { désireuses d'aller } \\
\text { a la confrontation }\end{array}$ & $\begin{array}{l}\text { - il n'y a pas de temps } \\
\text { disponible pour la } \\
\text { confrontation } \\
\text { - les parties prenantes } \\
\text { sont inaptes et/ou ne } \\
\text { désirent pas aller à la } \\
\text { confrontation }\end{array}$ \\
\hline
\end{tabular}

\section{BIBLIOGRAPHIE}

Afnor, Management de projet, Afnor, Paris, 1994. 
BARKI H., HARTWICK J., Measuring User Participation, User Involvment, and User Attitude MIS Quaterly, March 1994, pp. 59-79.

BRYNJOLfSSON E., The Productivity Paradox of Information Technology, Communications of the ACM, vol. 35, December 1993, pp. 66-77.

BRYNJOLFSSON E., Information, Assets, Technology and Organization, Management Science, vol. 40,N • 12, December 1994 pp. 1645-1662.

Kezsbom D.S., Schilling D.L., EdWARD K.A., Dynamic Project Management, « Wiley-Interscience John Wiley \& Sons Inc., 1989.

CAMERON K.S., Effectiveness as a Paradox : Consensus and Conflict of Organizationnal Effectiveness, Management Science, vol. 32, $\mathrm{N}^{\circ}$ 5, May 1986, pp. 539-553.

DeLone W., McLeAn E., Information Systems Success : The Quest of the Dependent Variable, Information Systems Research, vol. 3, March 1992, pp. 60-95.

KolbB D.M., BARTUneK J.M., Hidden Conflict in Organizations, Sage Publications, 1992.

LEWIN A.Y., MinTon J.W., Determining Organizational Effectiveness : another look, and an agenda for research, Management Science, vol. 29, N 5, May 1986, pp. 514-538.

MAHMOOD M., MANN G., Measuring the Impact of Information Technology on Organizational Performance : a Key Ratios Approach, in Proceedings of the Twenty-fourth International Conference on Systems Sciences, 1991, pp. 251-258, Los Alamitos, California.

MARCLNIAK R., Les Mesures de l'efficacité des projets informatiques : modélisation et validations, Doctorat en Sciences de gestion, Juin 1991, Université d'Aix-Marseille 3, I.A.E. Aix en Provence.

MARCINIAK R., Management des projets informatiques : complexité et gestion des conflits, Systèmes d'Information et Management, $\mathrm{n}^{\circ}$ 1, vol. 1, mars 1996 pp. 27-50.

MARCINIAK R., ROWE F, Systèmes d'information, dynamique et organisation, Economica collection PIQ, 1997.

Montebello M., Efficacité de l'entreprise : analyse et perspectives, Thèse pour le Doctorat d'Etat ès Sciences de Gestion, Université d'Aix-Marseille 3, IAE, 1976.

MORIN E., Introduction à la pensée complexe, Communication \& complexité, ESF, 1990.

NisSEN M., Valuing IT through Virtual Process Measurement, in Proceedings International Conference on Information Systems, December 1994, pp. 309-323, Vancouver, British Columbia.

QUINN R.E., RoHRBAUgh J., A Spatial Model of Effectiveness Criteria : Towards a Competing Values Approach to Organizational Analysis, Management Science, vol. 29, № 3, March 1983, pp. 363-377.

RoWE F., L'impact de l'informatisation sur la performance de l'entreprise, Revue française de gestion, $\mathrm{N}$ -97,1994, pp. 30-42.

ScoTT J., The Measurement of Information Systems Effectiveness : Evaluating a Measuring Instrument, in Proceedings International Conference on Information Systems, December 1994, pp. 111-128, Vancouver, British Columbia.

SENGE P., La cinquième discipline, First, 1991.

VAN DE Ven A.H., Ferry D., Measuring and Assessing Organizations, John Wiley \& Sons, 1980. 


\section{NOTES}

1. On entend par processus : une organisation de personnes, matières, énergie, équipements et procédés en activités conçues pour produire un résultat final spécifié.

2. Elle est largement illustrée dans les manuels par l'analogie des poupées gigognes

3. Pour une connaissance plus approfondie des fondements théoriques du modèle et de ses validations on se reportera a Marciniak K (1991 et 1996).

4. Environnement d'un système ouvert selon Monn, 1990 pp. 29-34

5. La structure dynamique du système projet, dévoile des processus amplificateurs, bénéfiques ou néfastes \{cf. 33).

\section{RÉSUMÉS}

Cet article propose une approche, inspirée des théories de la complexité, pour une meilleure compréhension du déroulement des projets. Cette approche a été soumise à une vérification empirique sur un échantillon de vingt quatre projets d'informatique de gestion et de mille répondants (utilisateurs, informaticiens, responsables utilisateurs et informaticiens). La gestion des conflits constitue le pivot du modèle, dans la mesure où l'ensemble des autres variables transitent par les modes de résolution des conflits.

An empirical and longitudinal study of software development projects was undertaken to investigate differents paradigms. Effectiveness is a multidimensional concept and operates as a paradox. Conflict management is important and conflict resolution can conduce to, or reduce effectiveness.

\section{AUTEUR}

\section{ROLANDE MARCINIAK}

Rolande Marciniak est Maître de conférences à l'Institut d'Informatique d'Entreprise (CNAM-IIE) et Secrétaire générale de l'AFITEP (Association francophone du management de projet). 